\title{
Supplementary material of
}

\section{"A procedure to clean, decompose and aggregate univariate time series"}

\section{Part I: Outliers}
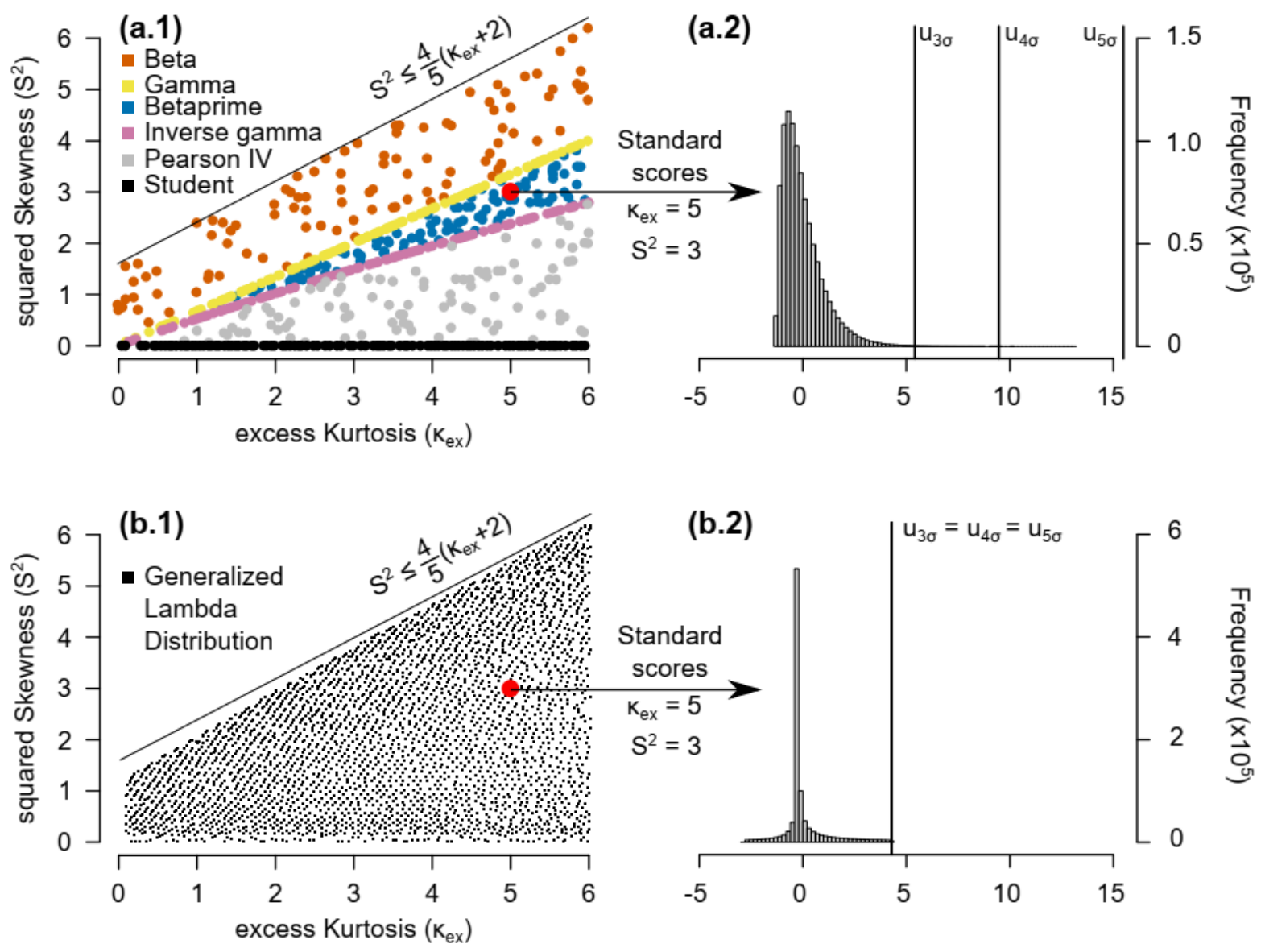

Fig. S1. Location of the 600 random distributions picked among the Pearson family in the $\left(\kappa_{e x}, S^{2}\right)$ space (panel a.1). Example of the three upper thresholds based on the boxplot rule $\left(u_{3 \sigma}, u_{4 \sigma}\right.$ and $\left.u_{5 \sigma}\right)$ to detect outliers for a Betaprime distribution with a skewness of 5 and a kurtosis of $\sqrt{3}$ (panel a.2). The Generalized Lambda Distribution (GLD) system is shown for comparison in the $\left(\kappa_{e x}, S^{2}\right)$ space (panel b.1). Similar figure than a.2 except for a distribution coming from the GLD system with a skewness of 5 and a kurtosis of $\sqrt{3}$ (panel b.2).

- Figure 1 shows that the Generalized Lambda Distribution (GLD) system produces non-realistic outlier thresholds because each distribution from this system is bounded. The Pearson family is therefore preferred over the GLD system to model outlier behaviors. 


\section{Part II: the past procedure}

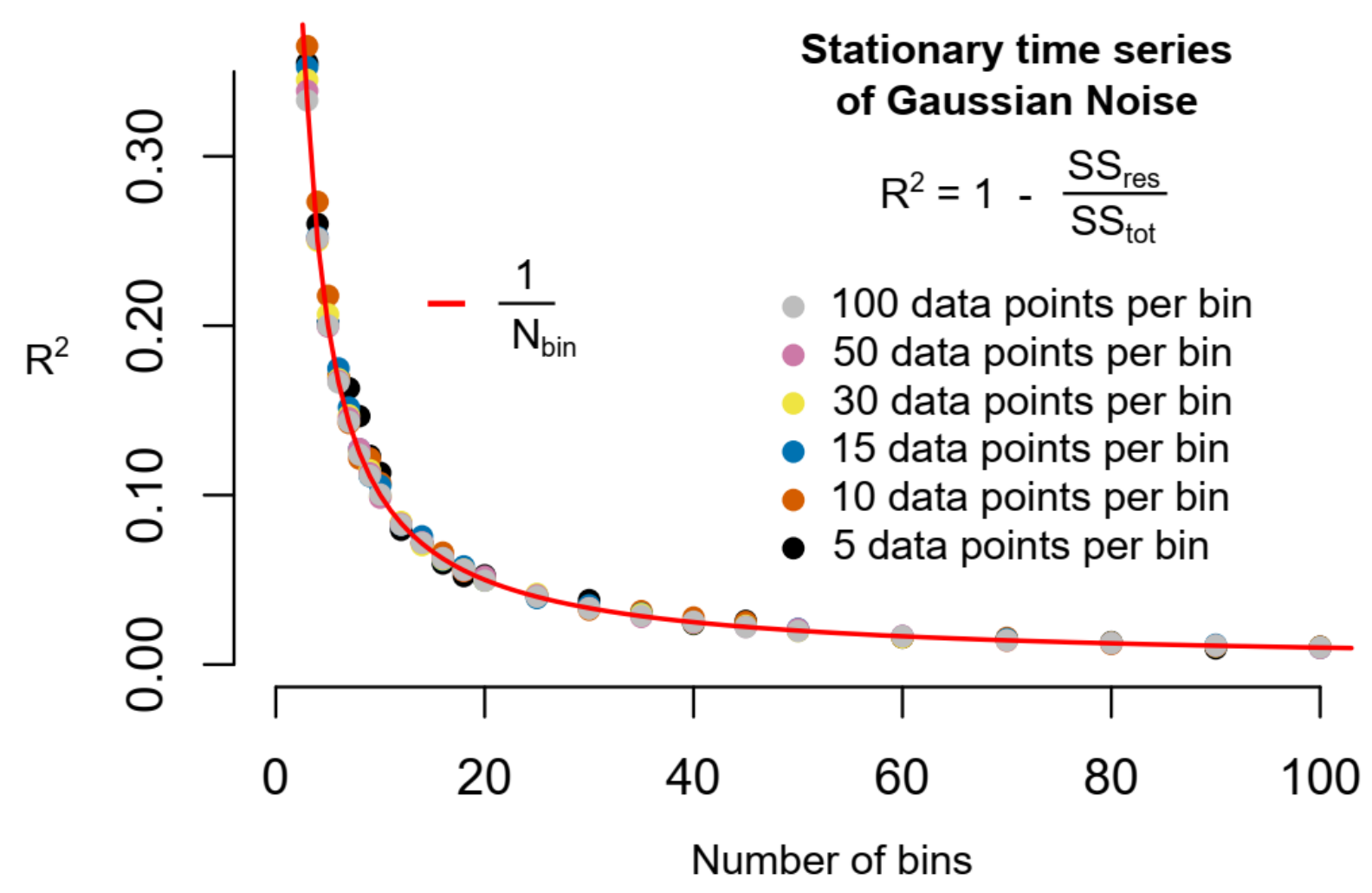

Fig. S2. The coefficient of determination $\left(R^{2}\right)$ has been calculated for multiple stationary time series of Gaussian noise $y$, with $S S_{\text {res }}=\sum\left(y_{i}-S_{i}\right)^{2}, S S_{\text {tot }}=\sum\left(y_{i}\right)^{2}$ and $S$ the cyclic component calculated with the past procedure.

- Considering Fig. S2, an inverse relationship appears between the coefficient of determination calculated on a pure Gaussian noise and the number of bin used (related to the sample size). This relationship is independent from the number of points per bin (illustrated by different colors). Theoretically, a stationary timeseries has a null cyclicity $\left(S=0, R^{2}=0\right)$. While this is observed for a large number of bins $\left(N_{b i n} \gg\right.$ 100), a bias of $N_{b i n}{ }^{-1}$ exists at a smaller amount and needs to be corrected. This justifies the definition of the stacked cycles index as $S C I=R^{2}-N_{b i n}^{-1}$. 